\title{
Clinical problems in the initiation and assessment of multidrug therapy
}

\author{
M F R WATERS, D S RIDLEY \& \\ MARIAN J RIDLEY \\ Hospital for Tropical Diseases, London NW1 OPE
}

\section{Introduction}

The chemotherapy of leprosy needs both to be as effective and also, certainly under field conditions, as safe, cheap, short, acceptable and easy to apply as possible. Now, 4 years after the introduction of the WHO Study Group regimens ${ }^{1}$ is a very appropriate moment to review progress in multidrug therapy (MDT).

The possibility of MDT of limited duration was pioneered by Professor Freerksen and his colleagues in Malta, ${ }^{2}$ at the time when the WHO 4th Expert Committee ${ }^{3}$ had just extended the recommended duration of dapsone monotherapy in lepromatous leprosy (LL) to ' 10 years after achieving inactivity', i.e. smear negativity. This entailed a total duration of about 20 years of dapsone in severe LL cases, although with the increasing number of relapses then being seen, many due to dapsone resistance, most leprologists were recommending that LL patients should be treated for life. Borderline-tuberculoid (BT) patients were usually treated for around 4-5 years; the time of achieving clinical inactivity was often difficult to determine and the bacteriological end-point, essential for assessing the effectiveness of chemotherapy, was not differentiated from the ending of immunological activity.

In the 1983 assessment of the small and mixed group of Maltese multibacillary leprosy (MBL) patients (mixed because most had received various durations of dapsone therapy before MDT and their sensitivities to dapsone were not investigated), Jopling ${ }^{4}$ found no case of relapse among $116 \mathrm{MBL}$ patients studied 5-108 months after stopping MDT. The significance of the 'solid' bacilli detected in a few smears was uncertain and warranted further investigation, although the ultimate and significant test of chemotherapeutic effectiveness is and will be the relapse rate. Dr Jopling's update on these selected smear positive patients, ${ }^{5}$ finding no relapses in April 1986, is therefore of special interest.

The second series of data supporting limited duration treatment has been known for some years, but unfortunately is only now in press. In Malaysia, we 
studied $362 \mathrm{LL}$ and BL patients who commenced treatment as inpatients with sulphones (the majority with dapsone) between 1948 and the end of 1951 . Therefore all may be assumed to have been dapsone sensitive. They all received dapsone 300-400 mg twice weekly by injection from around 1950 until 1963 and every dose was recorded. Then many were changed to oral dapsone. All stopped treatment in July 1970. A nine-year follow-up revealed a steady relapse rate of about $1 \%$ per annum. Among those relapsed patients who permitted further study, about half were found to have relapsed with various levels of dapsone resistance and half with dapsone sensitive stains of Mycobacterium leprae. This suggested that if all drug-resistant mutants could be eliminated by combined chemotherapy, the relapse rate from microbial persistence might well be acceptably low. ${ }^{6}$

In paucibacillary leprosy (PBL), two groups of workers showed that ultrashort course treatment with rifampicin was fully effective. Pattyn ${ }^{7}$ with Warndorff in Ethiopia and Bourland in Burundi showed that 8 weekly doses of 900-mg rifampicin cured tuberculoid leprosy; no relapses occurred in a follow-up period of 3 years. In the Philippines, Shepard ${ }^{8}$ showed that in tuberculoid (TT) patients given rifampicin $600 \mathrm{mg}$ daily for 14 days and BT patients for 21 days plus, in both groups, two injections of acedapsone, none relapsed during an average follow-up of 2 years, whereas similar groups of patients treated with dapsone for 1 year had a significant relapse rate.

It was on the basis of these four groups of data that the WHO Study Group ${ }^{1}$ introduced its recommendations, dividing leprosy patients into two groups, MBL including all $\mathrm{LL}, \mathrm{BL}$, and $\mathrm{BB}$ patients plus those $\mathrm{BT}$ patients with a bacterial index (BI) in smears (when untreated) at any site of $2+$ or more, and PBL being TT, BT and indeterminate with no smear site BI (when untreated) greater than $1+$. It was assumed that no PBL group patient would have a viable $M$. leprae population greater than $1 \times 10^{6}$ and would therefore be unlikely to harbour rifampicin-resistant mutants.

These recommendations, whether unchanged or with modifications, have now been applied in many parts of the world. In our own practice, and in discussions with many colleagues, we find three major problems equally applicable to Freerksen's and to the WHO regimens.

\section{Accuracy of classification}

Under field conditions, classification depends on both clinical and bacteriological assessment. Both can be a source of difficulty.

\section{a Bacterial}

The sites for slit-skin smears may be ill-chosen. The remedy lies in better training 
of those responsible for taking smears. But both adequate privacy and lighting are essential if the best sites are to be detected.

In the laboratory, smear staining must be checked. We have known two good laboratories to experience difficulty, both because of minor misunderstandings over staining techniques, one from under- and the other from over-decolourization. Smear technicians deserve our interest and encouragement. Smear reading and scoring should be standardized. ${ }^{9}$

\section{$b$ Clinical}

Patients diagnosed when downgrading from BT towards BL are often difficult to classify as recognizable changes in clinical appearances may not occur until several months after the bacterial load has begun to increase, and the histological classification to shift significantly. The skin smear results, however, should indicate the correct classification of MBL.

Neural leprosy, although nearly always BT or TT, may also occasionally cause problems, as nerve biopsy is seldom possible under field conditions, and even lepromin testing is not routinely performed in most centres. The following brief case histories (f ull reports to be published elsewhere) of two unusual patients illustrate the difficulties that may be encountered.

\section{Case 1.}

A 50-year-old unhappy Bangladeshi lady was referred in February 1980, complaining of numbness. She had been seen frequently by doctors over the previous two years suffering from numerous complaints, but in 1978 had been thought to be anaesthetic over the right hand and wrist. On examination, she was found to have hysterical anaesthesia over most of the body, including the axillae and ante-cubital fossae areas. No skin lesions were visible. But the right superficial radial nerve was significantly though only mildly thickened. She was assumed to have suffered from tuberculoid leprosy in her native country, which had either been treated or which had resolved spontaneously. Biopsy of the enlarged nerve, however, revealed active BL leprosy histologically, and the BI was $5+$ (Ridley scale). Skin smears from seven sites (both ear lobes and five random sites) were all negative for acid-fast bacilli (AFB).

\section{Case 2.}

This 15-year-old Vietnamese boy was ref erred in January, 1983. He gave a history of having developed numbness followed by weakness of his right leg and foot some 2 to 3 years earlier. Subsequently he developed numbness of the left leg, and more recently of his fingers. Three months before admission he developed a left foot drop. On examination, no specific skin lesions could be detected. He had 
bilateral foot drop, more marked on the right side, and an anaesthetic ulcer was present on the right sole. Most of the nerves of predilection in leprosy were enlarged, and the right ulnar nerve was tender. Skin smears from both ear lobes and four other sites were all negative for AFB. Nerve biopsy (Dr T L Pathi) from the left great auricular nerve area revealed a lymph node whose architecture was destroyed by massive infiltration of activated macrophages, containing 4+ AFB. The nerve was similarly destroyed and in addition to the macrophages or epithelioid cells, there were some lymphocytes. It was considered that although the precise classification was uncertain, the histological diagnosis was 'probably $\mathrm{BB}$, possibly BL'. The Mitsuda reaction measured $3 \mathrm{~mm}$ at 4 weeks. Taking all the data into account, he was classified as BB leprosy of the polyneuritic variety.

Under field conditions, both these patients would have been diagnosed as PBL, whereas nerve biopsy revealed that both were in fact MBL, and on WHO criteria should receive treatment for two years with three drugs.

\section{Differential diagnosis of relapse and reversal reaction in PBL}

Although the word 'relapse' in leprosy can be used in a number of different senses, when antimicrobial chemotherapy is under consideration it signifies the fresh multiplication (and spread) of surviving leprosy bacilli in a patient who had previously responded to therapy. On dapsone monotherapy, such bacteriological relapse was most frequently due to the development of drug resistance, but the MDT regimens have been designed to prevent the selection of drug resistant mutants of $M$. leprae. Of much greater concern now is the possibility of relapse due to the multiplication of persisting, drug-sensitive, physiologically-dormant bacilli occurring after the prescribed course of MDT has been completed.

Relapse in MBL is usually easy to diagnose from the increase in the $\mathrm{BI}$, the finding of new skin lesions (in LL leprosy, often of typical histoid appearance) with a high smear BI containing solid-staining bacilli, and the histological appearances. Bacilli obtained from a new lesion will multiply in the footpads of mice, and the drug sensitivities of the strain of $M$. leprae can be studied.

Relapse in PBL, is much more difficult to diagnose as the appearances often resemble closely, and may be indistinguishable from, those of reversal (upgrading, Jopling type 1) reactions. Indeed, the development of a reversal reaction may occasionally be the first sign of a relapse in BT leprosy. Pandian ${ }^{10}$ has recently listed six signs of relapse in PBL, namely the development of: erythema, thickening (infiltration) of skin lesions, new skin lesions, pain and tenderness in nerves, new muscle paralysis, and the extension (increase in diameter) of existing lesions. We have seen all these signs, save the last, in reversal reactions occurring in PBL patients on chemotherapy. But unless records are good, the increase in the size of a skin lesion would probably go undetected - who routinely measures and records the size (diameter) of tuberculoid skin lesions? Moreover, Leiker 
(personal communication) considers that extension may very rarely occur in reversal reaction unassociated with relapse. The histological appearances of new skin lesions also may fail to distinguish between relapse and reversal reaction. The development of bacterial positivity, with a rising BI in the smears, is most indicative of relapse provided that chance sampling variation can be excluded, but may not occur in tuberculoid relapse, and if it does, it may not always be detected at a very early stage.

Therefore early relapse may be very difficult if not impossible to distinguish from a mild reversal reaction, unless there is an undoubted increase in the size of the skin lesions, or an undoubted, significant increase in the smear BI. THELEP discussed the timing of the onset of the 'relapse/reaction', ${ }^{11}$ suggesting that signs developed within a year of diagnosis, i.e. within 6 months of stopping WHO PBL MDT, were almost certainly due to reversal reaction, whereas signs developed 18 months or more after diagnosis (or 12 months or more after stopping MDT) were more likely to be due to relapse. This could be a useful 'rule of thumb' in alerting clinicians to the possibility of relapse. But reversal reactions can undoubtedly if rarely occur a full three years after commencing effective chemotherapy in BT leprosy.

\section{Case 3.}

A 22-year-old East African Asian was seen in consultation in May 1980. He had doubtful very faint hypopigmented macules over much of his limbs. There was widespread enlargement of the nerves of predilection. Smears from six sites were all negative for AFB. Biopsy of the left superficial radial nerve revealed a light infiltrate of lymphocytes but no granuloma cells; AFB were present. $\mathrm{He}$ was treated with rifampicin $750 \mathrm{mg}$ daily for 17 days, and with dapsone. After 7 months he developed neuritis, but the then undoubted hypopigmented skin lesions did not develop signs of reversal reaction. He received steroids for 11 months. Throughout 1982, he was prescribed rifampicin $600 \mathrm{mg}$ daily on the first 2 days of each month plus daily dapsone. In March 1983, he developed symptoms of paraesthesiae and anxiety but there was no nerve tenderness. He was given clofazimine and the rifampicin and dapsone were continued. His Mitsuda reaction measured $5 \mathrm{~mm}$ at 21 days. In June 1983, 37 months after commencing therapy, during which time his urine was regularly positive for dapsone, he developed an acute right foot drop. On biopsy, the right sural nerve was found to be almost completely destroyed by an epithelioid granuloma, with lymphocytes and fibrosis. Fragmented or granular AFB $1+$ were present. The appearances were typical of BT leprosy, which was obviously active, though whether from reaction or relapse was uncertain histologically. In view of his chemotherapy record, and evidence of compliance, there is no doubt that this was a reversal reaction in the nerve.

Although this patient's case is very exceptional in our experience, it poses the 
question how long does it take for all granuloma and all AFB to be removed from severely affected BT nerves? A further sural nerve (left) biopsy at 52 months still showed an infiltrate of lymphocytes and fibroblasts in the perineurium and some perivascular lymphocytes within the nerve; although there was no granuloma or definite AFB, scanty AFB granules were still present.

Results to date suggest that short course MDT in PBL is very successful. Personal communications from doctors (Drs E S Thangaraj, P D Samson, A Thomas, L Hogerzeil, G Riedel, J Harris) working in three countries report only 19 'relapses' in over 3800 PBL patients followed for at least one year after stopping MDT; some of these 19 'relapses' may in fact have been reversal reactions. Much longer term follow up is essential, but the outlook appears very promising.

Reversal reaction may also occur late in MBL BL and BB patients. Case 1 developed a swollen right hand with median nerve neuritis, followed shortly by a near complete facial nerve paralysis associated with the development of a hypopigmented lesion (histologically BT) over the upper face and forehead, 56 months after commencing treatment. Under present guidelines she would have stopped treatment at 24 months, but for social reasons plus uncertainty concerning the length of treatment in 'neural' BL, MDT had not been stopped. Had it been, the differential diagnosis of the reversal reaction would have been very difficult especially as the appearance of new BT lesions may be the first sign of bacteriological relapse in dapsone-treated BL patients ${ }^{12}$ (Waters \& Ridley, unpublished).

\section{Histological differentiation of reaction and relapse}

Although the histological differentiation of reversal reaction and relapse in skin biopsy is regarded by some workers as difficult, we often find it to be of value. Reaction in mild or incipient cases, that may or may not yet be clinically apparent, is indicated by 1 , extracellular oedema in the collagen of the dermis, especially in the neighbourhood of the granuloma, and dilated lymphatics; or 2, a proliferation of fibrocytes (with small dark elongated muclei) throughout the dermis, even far removed from any granuloma. These two features may both be present. In more severe reactions the oedema causes disruption and dispersal of the granuloma in the acute phase. Later there is evidence of upgrading as the granuloma becomes reconstituted (downgrading is not likely to be the cause of a reaction after a period of intensive MDT).

There are 3 possible sources of confusion:

1 Relapse is sometimes associated with upgrading in the new lesion. ${ }^{12}$ The relapse lesion simply develops at a point higher up the spectrum than the former lesions, and is not associated with signs of reaction. However, this means that it 
may not be possible to distinguish a post-reaction lesion from relapse, and so it is advisable to select the most active-looking lesion for biopsy.

2 Relapse lesions are sometimes highly active, and in such cases there may be a localized proliferation of fibrocytes around the periphery of the granuloma. This can be confusing, especially if there is not much normal dermis in the biopsy. A moderate-sized specimen is therefore helpful.

3 In nerves an element of reaction is very common, whether or not there is a generalized rection clinically or histologically, and there is no sharp line of demarcation between activity and reaction. ${ }^{13}$ Nerve biopsies, as in case 3 , are less helpful therefore for this purpose than skin biopsies.

\section{Choice of chemotherapy and its duration}

There is no disagreement among scientists either over the principles of MDT or of its objectives, namely to overcome dapsone resistance, to prevent the emergence of new drug resistances, especially to rifampicin, and to cure bacteriologically the great majority of all leprosy patients with acceptable short-course chemotherapy. Nevertheless, there is considerable uncertainty concerning the choice of the third drug to accompany rifampicin and dapsone and over the duration of treatment. Freerksen has preferred prothionamide ${ }^{2}$ whereas $\mathrm{WHO}^{1}$ recommended using the less rapidly bactericidal clofazimine as safer under field conditions, although advising that a thiamide should be prescribed for those patients who rejected clofazimine because of the latter's effect on skin colour.

What is essential today is to assess the toxicity of prothionamide and ethionamide under leprosy control conditions. Among patients using Freerksen's regimen, Jopling ${ }^{4}$ has reported one case of jaundice and one case of significantly raised liver transaminases (LFT) in Malta (where monthly estimates of LFT were first introduced in 1979) from $122 \mathrm{MBL}$ patients. Alvarenga et al. ${ }^{14}$ reported 16 cases of jaundice among 754 patients, both MBL and PBL, receiving Isoprodian (12 of the 16 were also receiving rifampicin) in Paraguay. Much higher incidences of jaundice on combined rifampicin and thioamide treatment have been reported from China ${ }^{15}$ and Singapore ${ }^{16}$ in which countries clofazimine is not readily accepted, and from France and the French Caribbean. ${ }^{17,18}$ Therefore the possibility that genetic and environmental factors influence the incidence of toxic jaundice requires investigation.

In London since 1980 we have treated 37 patients with triple-drug therapy including a thioamide and five have developed jaundice. Two of these were previously untreated lepromatous patients, who commenced treatment in 1980 with rif ampicin $600 \mathrm{mg}$ daily plus ethionamide $500 \mathrm{mg}$ and dapsone $100 \mathrm{mg}$ daily. One, a Sudanese, became mildly jaundiced after 28 days, the other, an East African Punjabi, after 12 weeks. The three other patients were long-term dapsone-treated MBL patients who were prescribed a two-year course of 
rif ampicin $600 \mathrm{mg}$ daily on two consecutive days of each month, ethionamide 250 $\mathrm{mg}$ daily and dapsone either $50 \mathrm{mg}$ or $100 \mathrm{mg}$ daily. One was a Cypriot, another an Anglo-Indian and the third a Bengali. Jaundice developed between 2 and $4 \frac{1}{2}$ months of commencing triple-drug therapy. In no case was the jaundice severe, and four were subsequently restarted safely on rifampicin, but not on a thioamide. In no case was there any serological evidence of Hepatitis-B virus infection. But two patients did have marginally raised LFT levels before commencing the combined rifampicin-thioamide therapy, and we would now consider the finding of any LFT abnormality, however slight, a contraindication. Only one of the 37 patients studied was Chinese, and one Vietnamese. But in view of the different racial backgrounds of our five jaundiced patients, we have no evidence of a genetic factor. Although this is only a small series, its incidence of toxic hepatitis of $13.5 \%$, very similar to the incidence of $13 \%$ reported by Cartel et al.,${ }^{17}$ is distressing, and is in marked contrast to that of $1.6 \%$ in Malta, ${ }^{4}$ of $2 \cdot 1 \%$ in Paraguay, ${ }^{14}$ or of $4.5 \%$ reported by Pattyn and his colleagues. ${ }^{19}$ Further studies appear imperative.

Our limited experience in London does not yet provide any further evidence concerning the duration of treatment required to cure the different types of leprosy; this problem is addressed by other members of the Symposium. Longterm follow-up is essential, with careful investigation of every apparent relapse off treatment, if reliable data are to be obtained.

\section{Conclusions}

Although the majority of leprosy patients can be easily allocated to either PBL or to MBL, some may be very difficult, if not impossible, to classify under field control conditions, with resulting uncertainty over the type and duration of treatment required. Good standards of smear taking, staining and reading are essential in all leprosy treatment and control schemes, and scoring of smears should be standardized.

It is often very difficult, if not impossible at least in the short term, to distinguish between reversal reactions and bacteriological relapse off treatment in PBL patients who have completed their course of MDT. All 'relapse' patients require the most careful and thorough investigation if accurate assessment of the results of MDT are to be obtained. The definition of 'relapse' given by the WHO Study Group on the Epidemiology of Leprosy in Relation to Control ${ }^{20}$ fails to distinguish between these two groups and is therefore of no help in assessing bacteriological cure rates. Similar failure in the past to distinguish between bacterial relapse and late reversal reaction must cast doubts on many of the previous reports of relapse after completing treatment in tuberculoid leprosy.

Finally, although the basic principles of MDT are generally agreed, we still have insufficient data to decide, on balance, which is the best drug combination 
and the duration for which it should be given, to obtain the highest possible cure rates, in the shortest possible time, at the lowest risk of toxic side-effects under leprosy control conditions.

\section{References}

1 WHO Study Group. Chemotherapy of leprosy for control programmes. WHO Technical Report Ser No. 675. WHO Geneva, 1982.

2 Freerksen E, Rosenfeld M. Leprosy eradication project of Malta. Chemotherapy (Basel), 1977; 23: $356-386$.

3 World Health Organization Expert Committee on Leprosy 4th Report 1970. WHO Technical Report Ser No. 459. WHO Geneva, 1970.

${ }^{4}$ Jopling WH, Ridley MJ, Bonnice E, Depasquale G. A follow-up investigation of the Malta Project. Lepr Rev, 1984; 55: 247-253.

5 Jopling WH. A follow-up investigation of the Malta-Project, 1983 and 1986. Lepr Rev, in press.

${ }^{6}$ Waters MFR, Rees RJW, Laing ABG, Khoo KF, Meade TW, Parikshak N, North WRS. The rate of relapse in lepromatous leprosy following completion of twenty years of supervised sulphone therapy. Lepr Rev, 1986; 57: 101-9.

7 Warndorff J, Bourland J, Pattyn SR. Follow-up on short course two months rifampicin treatment of paucibacillary leprosy. Lepr Rev, 1982; 53: 9-17.

8 WHO Report of the Third Meeting of the Scientific Working Group on the Chemotherapy of Leprosy, Geneva, 20-22 October, 1980. Unpublished WHO document TDR/THELEP-SWG (3)/80.3.

${ }_{9}$ De Rijk AJ, Nilsson T, Chonde M. Quality control of skin smear services in leprosy programmes: preliminary experience with inter-observer comparison in routine services. Lepr Rev, 1985; 56: 177-191.

10 Pandian TD, Sithambaram M, Bharathi R, Ramu G. A study of relapse in non-lepromatous and intermediate groups of leprosy. Indian J Leprosy, 1985; 57: 149-158.

11 World Health Organization. Standard protocol for chemotherapy trials in non-lepromatous leprosy. WHO Geneva, 1982. Document TDR/THELEP/PROTOCOL/82.1.

12 Waters MFR, Ridley DS. Tuberculoid relapse in lepromatous leprosy. Int J Lepr, 1979; 47: 350.

13 Pearson JMH, Ross W.F. Nerve involvement in leprosy-Pathology, differential diagnosis and principles of management. Lepr Rev, 1975; 46: 199-212.

14 Alvarenga A, Leguizamon O, Frutos V, Graf von Ballestrem W. The leprosy eradication programme in Paraguay with the combination rifampicin-Isoprodian. Int J Lepr, 1984; 52: 714.

15 Ji Baohong, Chen Jiakun, Wang Chenmin, Xia Guang. Hepatotoxicity of combined therapy with rifampicin and daily prothionamide for leprosy. Lepr Rev, 1984; 55: 283-289.

16 Country Report on Experiences in MDT. Singapore. WHO interregional workshop on multidrug therapy regimens for leprosy control, Manila. 25-29 October 1984. Unpublished WHO document WPR/LEP/INF./26.

17 Cartel J-L, Millan J, Guelpa-Lauras, C-C, Grosset JH. Hepatitis in leprosy patients treated by a daily combination of dapsone, rifampin and a thioamide. Int $J$ Lepr, 1983; 51: 461-465.

${ }_{18}$ Cartel J-L, Naudillon Y, Artus J-C, Grosset JH. Hepatotoxicity of the daily combination of $5 \mathrm{mg} / \mathrm{kg}$ prothionamide $+10 \mathrm{mg} / \mathrm{kg}$ rifampin. Int J Lepr, 1985; 53: 15-18.

19 Pattyn SR, Janssens L, Bourland J, Saylan T, Davies EM, Grillone S, Feracci C, and the Collaborative Study Group for the Treatment of Leprosy. Hepatotoxicity of the combination of rifampin-ethionamide in the treatment of multibacillary leprosy. Int J Lepr, 1984; 52: 1-6.

${ }^{20}$ WHO Study Group. Epidemiology of leprosy in relation to control. WHO Technical Report Ser No. 716. WHO Geneva, 1985. 